\title{
Efeito da Densidade e de Programas de Alimentação sobre o Desempenho de Frangos de Corte
}

\section{Geraldo Roberto Quintão Lana', René Geraldo Cordeiro Silva Junior², Sandra Roseli Valerio ${ }^{3}$, Ângela Maria Quintão Lana ${ }^{4}$, Elísia Carmem G. Bastos Cordeiro ${ }^{2}$}

\begin{abstract}
RESUMO - O objetivo deste experimento foi avaliar o efeito da densidade e de programas de alimentação sobre o desempenho produtivo e o rendimento de carcaça e de cortes nobres em frangos de corte aos 42 dias de idade. O delineamento experimental foi o inteiramente casualizado, num esquema fatorial $3 \times 2$ (densidades, programas de alimentação), com quatro repetições cada. As densidades foram: 10, 12 e 16 aves $/ \mathrm{m}^{2}$. A alimentação foi constituída de programa 1 (quatro rações): pré-inicial, inicial, crescimento e final; e programa 2 (cinco rações): pré-inicial I, pré-inicial II, inicial, crescimento e final. Na fase de 1 a 7 dias houve diferença significativa $(\mathrm{P}<0,05)$ entre densidades para consumo de ração, ganho de peso e conversão alimentar. Para a fase de 15 a 21 e 22 a 28 dias de idade, os melhores resultados de ganho de peso e conversão alimentar foram obtidos na densidade de 16 aves $/ \mathrm{m}^{2}$. Nas fases de 8 a 14,29 a 35 e 36 a 42 dias de idade e no período total, não foram observadas diferenças significativas entre os programas de alimentação e entre densidades em nenhum dos parâmetros avaliados. Recomenda-se para frangos de corte, machos, de 1 a 42 dias de idade, a densidade de 16 aves $/ \mathrm{m}^{2}$, independentemente do programa de alimentação.
\end{abstract}

Palavras-chave: densidade, desempenho, frango de corte, programa de alimentação, rendimento de carcaça e cortes nobres

\section{Effect of Density and Feeding Programs on Broiler Chickens Performance}

\begin{abstract}
The objective of this work was to evaluate the effects of density and feeding programs on the productive performance and carcass and prime cuts yield in broiler chickens at 42 days of age. A completely randomized design, in a 3x2 (density x feeding programs) factorial scheme, with three replications, was used. The densities were: 10,12 and 16 birds $/ \mathrm{m}^{2}$. The feeding was composed of program 1 (four diets): pre-initial, initial, growing and finishing and program 2 (five diets): pre-initial I, pre-initial II, initial, growing and finishing. In the phase from 1 to 7 days there was significant difference among densities for feed intake, weight gain and feed:gain ratio. For the phase from 15 to 21 and 22 to 28 days the better results of weight gain and feed:gain were obtained in the density of 16 aves $/ \mathrm{m}^{2}$. No significant differences were observed for the phases from 8 to 14,29 to 35 and 36 to 42 days and total period of age among the feeding programs and between densities for all evaluated parameters. It is recommended for males broiler chickens, from 1 to 42 days old, the density of 16 aves $/ \mathrm{m}^{2}$, independent of the feeding program.
\end{abstract}

Key Words: broiler-type chicken, density, feeding program, performance, carcass and prime cuts yield

\section{Introdução}

A avicultura é uma das atividades de produção animal que mais se desenvolveram nos últimos anos. Isso se deve, basicamente, à busca de novos sistemas de criação, que objetivam a maior produtividade no menor tempo possível. Um desses sistemas é a criação de frangos em alta densidade, o que, muitas vezes dá origem a frangos com menor peso ao abate, podendo ao mesmo tempo promover aumento da remuneração dos produtores (LUCCHESE FILHO, 1997; MUNARI, 1997). O aumento da densidade populacional propicia algumas desvantagens, como a pior qualidade da carcaça, a alteração da ordem social e as piores condições atmosféricas do galpão (SIMON, 1997). Para conseguir resultados positivos com esse sistema, os produtores devem realizar rigoroso planejamento (ABREU, 1999).

A crescente pressão para redução dos custos na criação de frangos de corte, aliada ao alto custo com a alimentação e aos baixos preços pagos pelo frango vivo, têm levado uma série de empresas e criadores a elevar a taxa de lotação, como forma de reduzir os custos de mão-de-obra e de investimentos em novos aviários. No entanto, na maioria das vezes, isso tem sido feito sem a necessária readequação de equipa-

\footnotetext{
1 Professor do DZ/UFRPE. Rua Dom Manoel de Medeiros, s/n - Dois Irmãos. Recife - PE. CEP: 52.171-900. E.mail: glana@econnet.com.br

2 Méd. Veterinário(a). Doutorando(a) do DZ/UFRPE - Recife - PE. E.mail: rjr@elogica.com.br

3 Zootecnista. Doutoranda do DZO/UFV. Viçosa - MG. E.mail: svalerio@alunos.ufv.br

4 Professora do Instituto de Ciências Exatas da UFRRJ - Seropédica, RJ.
} 


\section{LANA et al.}

mentos, nutrição e manejo ambiental à nova situação.

O objetivo da presente pesquisa foi verificar o efeito de programas de alimentação e da densidade sobre o desempenho produtivo e o rendimento de carcaça e corte nobres (peito, coxas e sobrecoxas) de frangos de corte.

\section{Material e Métodos}

O experimento foi realizado no setor de Avicultura do Departamento de Zootecnia da Universidade Federal Rural de Pernambuco - UFRPE, Recife - PE.

$\mathrm{O}$ galpão, orientado no sentido leste-oeste, era coberto com telhas onduladas de cimento-amianto e possuía piso cimentado e paredes laterais com $0,80 \mathrm{~m}$ de altura, completadas com telas de arame 18 e malha de uma polegada. Foram instaladas ao redor do galpão cortinas de polietileno, com a finalidade de oferecer às aves o máximo conforto. A parte interna do galpão era constituída por 24 boxes com área de $1,0 \mathrm{~m}^{2}$; cada box continha uma lâmpada incandescente de $100 \mathrm{w}$, utilizada como fonte de aquecimento durante os 10 primeiros dias de vida das aves.

Foram utilizados trezentos e quatro pintos de um dia de idade, machos, da marca comercial Hubbard, com peso médio inicial de 49,6 g, vacinados contra as doenças de Newcastle, cepa HB1, via ocular, e com vacina de Marek, via subcutânea. As aves foram criadas em piso com cama de maravalha, a uma espessura de $5,0 \mathrm{~cm}$. Nos 10 primeiros dias, foram utilizados lâmpadas de infravermelho como fonte de aquecimento, bebedouros tipo pressão e comedouros tipo bandeja, sendo substituídos gradativamente por bebedouros pendulares e comedouros tubulares.

Nos três primeiros dias de idade, adicionou-se à água um suplemento de vitaminas, aminoácidos e eletrólitos, obedecendo às recomendações do fabricante. $\mathrm{O}$ fornecimento de ração e água foi à vontade, durante todo o período experimental. O manejo dos equipamentos e das aves foi conduzido de acordo com o descrito por LANA (2000), em que a iluminação artificial foi fornecida a partir do primeiro dia de vida das aves com um fotoperíodo de 24 horas de luz.

As temperaturas máximas e mínimas no período experimental foram obtidas diariamente ( 9 horas), por meio de dois termômetros colocados à altura das aves, localizados em pontos médios da instalação (Tabela 1).

Foram utilizadas as densidades de 10,12 e 16 frangos $/ \mathrm{m}^{2}$ e dois programas de alimentação, constituídos por diferentes tipos de rações, de acordo com a fase de crescimento das aves, sendo: programa 1- pré-inicial I (1 a 14 dias), inicial (15 a 21 dias), crescimento (22 a 35 dias) e final (36 a 42 dias); e programa 2- pré-inicial I ( 1 a 7 dias), pré-inicial II (8 a 14 dias), inicial (15 a 21 dias), crescimento (22 a 35 dias) e final (36 a 42 dias). As rações à base de milho e farelo de soja foram formuladas para atender às exigências nutricionais preconizadas por ROSTAGNO et al. (1996), de acordo com os programas de alimentação e a fase de crescimento das aves (Tabela 2).

As 304 aves foram distribuídas nos seis tratamentos da seguinte forma: densidade de 10 aves $/ \mathrm{m}^{2}-40$ aves - nos programas 1 e 2 , perfazendo um total de 80 aves; densidade de 12 aves $/ \mathrm{m}^{2}$ - 48 aves - nos programas 1 e 2 , perfazendo um total de 96; aves e densidade de 16 aves $/ \mathrm{m}^{2}$ - 64 aves - nos programas 1 e 2 , perfazendo um total de 128 aves.

O delineamento experimental utilizado foi o inteiramente casualizado, em esquema fatorial 3x2 (densidades e programas de alimentação), com quatro repetições nas densidades de 10,12 e 16 frangos $/ \mathrm{m}^{2}$. As variáveis estudadas foram analisadas utilizandose o programa SAEG (Sistema para Análises Estatísticas), desenvolvido pela UFV (1982). As comparações entre as médias dos tratamentos foram realizadas, utilizando-se o teste de Tukey.

Os parâmetros avaliados foram o consumo de ração; o ganho de peso; a conversão alimentar de 1 a 7,8 a 14,15 a 21,22 a 28,29 a 35,36 a 42 e 1 a 42 dias de idade; os pesos ao abate; as produções em $\mathrm{kg} / \mathrm{PV} / \mathrm{m}^{2}$; os pesos de carcaças e de cortes nobres (peito, coxas e sobrecoxas); e os rendimentos de carcaças e cortes nobres aos 42 dias de idade.

Tabela 1 - Temperaturas médias máximas, mínimas e médias do ar durante o período experimental

Table 1 - Average air temperatures of maximum, minimum and medium during the experimental period

\begin{tabular}{lccc}
\hline \multirow{2}{*}{$\begin{array}{l}\text { Diass } \\
\text { Days }\end{array}$} & \multicolumn{3}{c}{$\begin{array}{c}\text { Temperaturas }\left({ }^{\circ} \mathrm{C}\right) \\
\text { Temperatures }\end{array}$} \\
\cline { 2 - 4 } & $\begin{array}{c}\text { Máxima } \\
\text { Maximum }\end{array}$ & $\begin{array}{c}\text { Mínima } \\
\text { Minimum }\end{array}$ & $\begin{array}{c}\text { Média } \\
\text { Average }\end{array}$ \\
\hline $1-7$ & 29,1 & 22,6 & 25,8 \\
$8-14$ & 29,3 & 22,8 & 26,0 \\
$15-21$ & 29,2 & 23,4 & 26,3 \\
$22-28$ & 29,1 & 23,5 & 26,3 \\
$29-35$ & 29,4 & 24,8 & 27,1 \\
$36-42$ & 30,2 & 25,2 & 27,7 \\
\hline
\end{tabular}


1260 Rev. bras. zootec.

Tabela 2 - Composição percentual das rações experimentais

Table 2 - Composition of experimental diets (\%)

\begin{tabular}{|c|c|c|c|c|c|}
\hline \multirow[t]{2}{*}{$\begin{array}{l}\text { Ingredientes } \\
\text { Ingredients }\end{array}$} & \multicolumn{5}{|c|}{$\begin{array}{c}\text { Rações experimentais } \\
\text { Experimental diets }\end{array}$} \\
\hline & $\begin{array}{c}\text { Pré-inicial I } \\
\text { Pre-initial I }\end{array}$ & $\begin{array}{l}\text { Pré-inicial II } \\
\text { Pre-initial II }\end{array}$ & $\begin{array}{c}\text { Inicial } \\
\text { Initial }\end{array}$ & $\begin{array}{c}\text { Crescimento } \\
\text { Growth }\end{array}$ & $\begin{array}{l}\text { Final } \\
\text { Final }\end{array}$ \\
\hline $\begin{array}{l}\text { Milho } \\
\text { Corn }\end{array}$ & 57,31 & 58,34 & 59,90 & 60,88 & 61,81 \\
\hline $\begin{array}{l}\text { Farelo de soja } \\
\text { Soybean meal }\end{array}$ & 38,38 & 35,91 & 33,52 & 31,12 & 28,77 \\
\hline $\begin{array}{l}\text { Fosfato bicálcico } \\
\text { Dicalcium phosphate }\end{array}$ & 1,78 & 1,80 & 1,66 & 1,67 & 1,69 \\
\hline $\begin{array}{l}\text { Óleo } \\
\text { Soybean oil }\end{array}$ & 0,63 & 1,99 & 3,17 & 4,54 & 5,93 \\
\hline $\begin{array}{l}\text { Calcário } \\
\text { Limestone }\end{array}$ & 1,15 & 1,16 & 1,00 & 1,02 & 1,03 \\
\hline $\begin{array}{l}\text { Sal } \\
\text { Salt }\end{array}$ & 0,27 & 0,27 & 0,27 & 0,28 & 0,29 \\
\hline $\begin{array}{l}\text { DL-Metionina } \\
\text { DL Methionine }\end{array}$ & 0,21 & 0,24 & 0,21 & 0,22 & 0,21 \\
\hline $\begin{array}{l}\mathrm{L} \text {-Lisina } \mathrm{HCl} \\
\text { L-Lysine } \mathrm{HCl}\end{array}$ & - & 0,02 & - & - & - \\
\hline $\begin{array}{l}\text { Bacitracina de zinco } \\
\text { Zinc bacitracin }\end{array}$ & 0,01 & 0,01 & 0,01 & 0,01 & 0,01 \\
\hline $\begin{array}{l}\text { BHT } \\
\text { Coxistac }\end{array}$ & $\begin{array}{l}0,01 \\
0,10\end{array}$ & $\begin{array}{l}0,01 \\
0,10\end{array}$ & $\begin{array}{l}0,01 \\
0,10\end{array}$ & $\begin{array}{l}0,01 \\
0,10\end{array}$ & $\begin{array}{l}0,01 \\
0,10\end{array}$ \\
\hline $\begin{array}{l}\text { Coccidiostatic } \\
\text { Premix } \\
\text { Calculado }\end{array}$ & 0,15 & 0,15 & 0,15 & 0,15 & 0,15 \\
\hline $\begin{array}{l}\text { Calculated } \\
\text { Energia metabolizável, kcal/kg } \\
\text { Metabolizable energy }\end{array}$ & 2900 & 3000 & 3100 & 3200 & 3300 \\
\hline $\begin{array}{l}\text { Proteína bruta, } \% \\
\text { Crude protein }\end{array}$ & 22,50 & 21,50 & 20,50 & 19,50 & 18,50 \\
\hline $\begin{array}{l}\text { Fósforo disponível, \% } \\
\text { Available phosphorus }\end{array}$ & 0,45 & 0,45 & 0,42 & 0,42 & 0,42 \\
\hline $\begin{array}{l}\text { Metionina, \% } \\
\text { Methionine }\end{array}$ & 0,55 & 0,57 & 0,53 & 0,52 & 0,50 \\
\hline $\begin{array}{l}\text { Metionina + cistina, } \% \\
\text { Methionine + cystine }\end{array}$ & 0,92 & 0,92 & 0,87 & 0,85 & 0,81 \\
\hline $\begin{array}{l}\mathrm{Ca}, \% \\
\text { Lisina, \% } \\
\text { Lysine }\end{array}$ & $\begin{array}{l}1,00 \\
1,23\end{array}$ & $\begin{array}{l}1,00 \\
1,18\end{array}$ & $\begin{array}{l}0,90 \\
1,10\end{array}$ & $\begin{array}{l}0,90 \\
1,03\end{array}$ & $\begin{array}{l}0,90 \\
0,97\end{array}$ \\
\hline 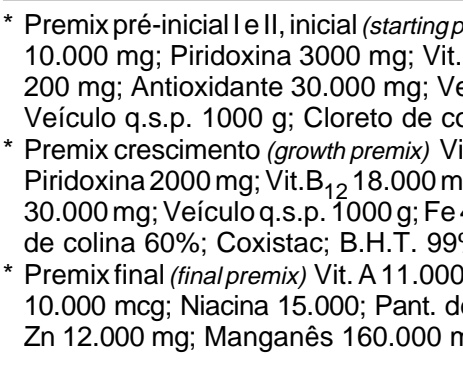 & $\begin{array}{l}\text { emix) Vit. A } 15.00 \\
31220.000 \text { mcg; } \\
\text { ículo q.s.p. } 100 \\
\text { lina } 60 \% \text {; Coxis } \\
\text { A } 12000000 U \text {; } \\
\text { g; Niacina } 33.00 \\
0.000 \text { mg; Cu12 } \\
0 \\
000 \text { Ul; Vit. } D_{3} 3 . \\
\text { cálcio } 10.000\end{array}$ & 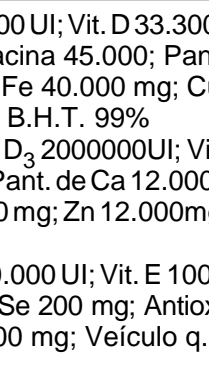 & $\begin{array}{l}\text { it. E20.00 } \\
\text { o } 15.000 \\
\mathrm{ng} ; \mathrm{Zn} 12 \\
\mathrm{mg} \text {; Vit.K } \\
\text { ó́lico } 10 \\
000 \mathrm{mg} ; \mathrm{C} \\
\mathrm{K} \\
000 \mathrm{mg} ;\end{array}$ & $\begin{array}{l}\mathrm{K}_{3} 3000 \mathrm{mg} \text {; Tiami } \\
\text { o fólico } 1350 \mathrm{mg} \text {; } \\
\text { Mn } 160.000 \mathrm{mg} \text {; } \\
\text { g; Tiamina } 2000 \mathrm{~m} \\
\text { otina } 30 \mathrm{mg} \text {; Selêr } \\
\text {; I } 2500 \mathrm{mg} \text {; Veícl } \\
\text { ina } 2000 \mathrm{mg} \text {; Ribo } \\
\text { s.p. } 1000 \mathrm{~g} ; \text { Fe } 40 \\
\text { a } 60 \% \text {; Coxistac; }\end{array}$ & $\begin{array}{l}\text { Riboflavina } \\
\text { ng; Selênio } \\
\text { I } 2500 \mathrm{mg} \text {; } \\
\text { la } 7500 \mathrm{mg} \text {; } \\
\text { ntioxidante } \\
0 \mathrm{~g} \text {; Cloreto } \\
\text { mg; Vit. } \mathrm{B}_{12} \\
12000 \mathrm{mg}\end{array}$ \\
\hline
\end{tabular}

No 42음 dia, todas as aves foram pesadas individualmente, sendo abatidas duas aves de cada boxe, de peso médio, após jejum de seis horas, e evisceradas, para determinação do rendimento de carcaça e cortes nobres, os quais foram calculados em relação ao peso ao abate.

\section{Resultados e Discussão}

Os resultados referentes ao consumo de ração semanal e total das aves no período de 1 a 42 dias de idade, de acordo com o programa de alimentação e as 
LANA et al.

Tabela 3 - Consumo de ração $(\mathrm{g})$ dos frangos nos períodos semanais e total (1 a 42 dias), de acordo com o programa de alimentação e a densidade

Table 3 - Feed intake $(g)$ of broiler chickens in the weekly and total periods (1 to 42 days), according to feeding program and density

\begin{tabular}{|c|c|c|c|c|c|}
\hline \multirow[t]{2}{*}{$\begin{array}{l}\text { Período (dias) } \\
\text { Period (days) }\end{array}$} & \multirow[t]{2}{*}{$\begin{array}{c}\text { Programa de alimentação } \\
\text { Feeding program }\end{array}$} & \multicolumn{3}{|c|}{$\begin{array}{c}\text { Densidades } \\
\text { Densities }\end{array}$} & \multirow[t]{2}{*}{$\begin{array}{l}\text { Média } \\
\text { Average }\end{array}$} \\
\hline & & 10 & 12 & 16 & \\
\hline \multirow[t]{2}{*}{$1-7$} & P1 & $176,4^{\mathrm{a}}$ & $157,9^{b}$ & $163,2^{b}$ & 172,7 \\
\hline & $\mathrm{P} 2$ & $173,2^{\mathrm{a}}$ & $160,2^{b}$ & $167,3^{b}$ & 166,9 \\
\hline \multirow[t]{2}{*}{$8-14$} & P1 & 397,9 & 390,3 & 381,4 & 389,9 \\
\hline & $\mathrm{P} 2$ & 388,7 & 391,5 & 385,7 & 388,6 \\
\hline \multirow[t]{2}{*}{$15-21$} & P1 & 662,3 & 641,8 & 642,3 & 648,8 \\
\hline & $\mathrm{P} 2$ & 664,9 & 642,4 & 674,2 & 660,5 \\
\hline \multirow{2}{*}{$22-28$} & $\mathrm{P} 1$ & 919,4 & 930,7 & 928,9 & 926,4 \\
\hline & $\mathrm{P} 2$ & 948,2 & 941,2 & 945,8 & 945,1 \\
\hline \multirow[t]{2}{*}{$29-35$} & P1 & 1163,4 & 1126,0 & 1149,4 & 1146,6 \\
\hline & P2 & 1180,1 & 1160,0 & 1125,9 & 1155,3 \\
\hline \multirow[t]{2}{*}{$36-42$} & P1 & 1133,8 & 1186,0 & 1258,1 & $1192,7^{\mathrm{A}}$ \\
\hline & $\mathrm{P} 2$ & $1347,0^{\mathrm{a}}$ & $1191,6^{\mathrm{b}}$ & $1234,0^{\mathrm{b}}$ & $1257,5^{\mathrm{B}}$ \\
\hline \multirow[t]{2}{*}{$1-42$} & $\mathrm{P} 1$ & 4453,2 & 4432,7 & 4523,3 & 4477,1 \\
\hline & $\mathrm{P} 2$ & 4702,1 & 4486,3 & 4532,9 & 4574,1 \\
\hline
\end{tabular}

Médias seguidas de letras diferentes, na mesma linha ou coluna, diferem (Tukey, $\mathrm{P}<0,05)$.

Means followed by different letters, in the same line or column, differ (Tukey, $P<.05)$.

densidades, encontram-se na Tabela 3. Não houve diferenças significativas $(\mathrm{P}>0,05)$ entre os programas de alimentação, assim como não foram constatadas interações entre programas de alimentação e densidades para o consumo de ração dos frangos de 1 a 42 dias de idade. Durante as fases de 1 a 7 e 36 a 42 dias de idade, observaram diferenças significativas $(\mathrm{P}<0,05)$ entre as densidades no que se refere ao consumo de ração das aves.

Os animais alojados à densidade de 10 aves $/ \mathrm{m}^{2}$ apresentaram maior consumo de ração $(176,4 \mathrm{~g})$, independentemente do programa de alimentação. Verificou-se interação significativa $(\mathrm{P}<0,05)$, na fase de 36 a 42 dias de idade, entre densidade e programa de alimentação para o consumo de ração, sendo que as aves do programa 2 (pré-inicial I, préinicial II, inicial , crescimento e final), submetidas a densidade de 10 aves $/ \mathrm{m}^{2}$, apresentaram maior consumo de ração (1347 g). Tal fato se deve, provavelmente, ao conforto ambiental, qualidade da cama e do ar, que tem influência direta no comportamento das aves, e, ainda ao melhor equilíbrio dos nutrientes, visto que o programa alimentar dispõe de maior número de rações.

Para as fases de 8 a 14,15 a 21,22 a 28 e 29 a 35 dias de idade, não se observaram diferenças significativas $(\mathrm{P}>0,05)$ entre programas de alimentação e entre densidades para consumo de ração dos frangos. As interações entre programas de alimentação e densidades também não foram significativas nessas fases.
No período total, ou seja, de 1 a 42 dias de idade, não ocorreram diferenças significativas $(\mathrm{P}>0,05)$ entre programas de alimentação e entre as densidades para consumo de ração. As interações entre programas de alimentação e densidades também não foram significativas. O maior consumo de ração $(4702,1 \mathrm{~g})$ foi observado no programa 2 na densidade de 10 aves $/ \mathrm{m}^{2}$.

Os resultados obtidos, neste estudo foram semelhantes aos descritos por SHIROMA et al. (1996) e STRINGHINI et al. (1997b, 1998), que avaliaram diferentes programas de alimentação e densidade e não verificaram diferenças significativas no consumo de ração durante o período total de criação. No entanto, os estudos realizados por HELLMEISTER FILHO et al. (1998) demonstraram diferenças significativas no consumo de ração dos frangos de corte criados em diferentes densidades.

$\mathrm{O}$ ganho de peso apresentado pelas aves no período de 1 a 42 dias, de acordo com o programa de alimentação e a densidade, encontram-se na Tabela 4. Observaram-se, na fase de 22 a 28 dias de idade, diferenças significativas $(\mathrm{P}<0,05)$ entre os programas de alimentação no ganho de peso. As interações entre programas de alimentação e densidades não foram significativas. Os ganhos de peso das aves foram de 530,0 e 550,8 g, para os programas 1 e 2 , respectivamente.

Nas fases de 1 a 7 e 15 a 21 dias de idade foram observadas diferenças significativas $(\mathrm{P}<0,05)$ entre as densidades. Os animais alojados sob a densidade 
1262 Rev. bras. zootec.

Tabela 4 - Ganho de peso (g) de frangos de corte nos períodos semanais e total (1 a 42 dias), de acordo com o programa de alimentação e a densidade

Table 4 - Weight gain $(g)$ of broiler chickens in the weekly and total periods (1 to 42 days), according to feeding program and density

\begin{tabular}{|c|c|c|c|c|c|}
\hline \multirow[t]{2}{*}{$\begin{array}{l}\text { Período (dias) } \\
\text { Period (days) }\end{array}$} & \multirow[t]{2}{*}{$\begin{array}{c}\text { Programa de alimentação } \\
\text { Feeding program }\end{array}$} & \multicolumn{3}{|c|}{$\begin{array}{c}\text { Densidades } \\
\text { Densities }\end{array}$} & \multirow[t]{2}{*}{$\begin{array}{l}\text { Média } \\
\text { Average }\end{array}$} \\
\hline & & 10 & 12 & 16 & \\
\hline \multirow[t]{2}{*}{$1-7$} & $\mathrm{P} 1$ & $109,3^{a}$ & $109,1^{\mathrm{a}}$ & $106,7^{b}$ & 108,4 \\
\hline & $\mathrm{P} 2$ & $101,0^{\mathrm{c}}$ & $107,4^{b}$ & $112,3^{\mathrm{a}}$ & 106,9 \\
\hline \multirow[t]{2}{*}{$8-14$} & P1 & 251,5 & 251,9 & 243,9 & 249,1 \\
\hline & $\mathrm{P} 2$ & 249,7 & 257,4 & 266,0 & 257,7 \\
\hline \multirow{2}{*}{$15-21$} & $\mathrm{P} 1$ & $446,1^{b}$ & $452,5^{b}$ & $455,4^{\mathrm{a}}$ & 451,3 \\
\hline & $\mathrm{P} 2$ & $450,0^{\mathrm{b}}$ & $444,3^{b}$ & $465,6^{\mathrm{a}}$ & 453,3 \\
\hline \multirow[t]{2}{*}{$22-28$} & $\mathrm{P} 1$ & 529,3 & 533,5 & 527,2 & $530,0^{\mathrm{A}}$ \\
\hline & $\mathrm{P} 2$ & 562,7 & 552,5 & 537,3 & $550,8^{\mathrm{B}}$ \\
\hline \multirow[t]{2}{*}{$29-35$} & $\mathrm{P} 1$ & 570,8 & 585,4 & 577,1 & 577,8 \\
\hline & $\mathrm{P} 2$ & 606,2 & 596,0 & 561,0 & 587,8 \\
\hline \multirow[t]{2}{*}{$36-42$} & $\mathrm{P} 1$ & 434,8 & 415,8 & 464,5 & 438,4 \\
\hline & $\mathrm{P} 2$ & 477,9 & 430,3 & 436,0 & 448,1 \\
\hline \multirow[t]{2}{*}{$1-42$} & $\mathrm{P} 1$ & 2341,8 & 2348,2 & 2374,8 & 2406,1 \\
\hline & $\mathrm{P} 2$ & 2447,5 & 2387,9 & 2378,2 & 2404,6 \\
\hline
\end{tabular}

Médias seguidas de letras diferentes, na mesma linha ou coluna, diferem (Tukey, $\mathrm{P}<0,05$ ).

Means followed by different letters, in the same line or column, differ (Tukey, $P<.05)$.

de 16 aves $/ \mathrm{m}^{2}$, submetida no programa de alimentação 2, apresentaram maior ganho de peso durante o período de 1 a 7 dias $(112,3 \mathrm{~g})$ e 15 a 21 dias de idade $(465,6 \mathrm{~g})$. O maior ganho de peso para as aves na densidade de 16 aves $/ \mathrm{m}^{2}$ se devem provavelmente ao melhor conforto proporcionado pela maior densidade na primeira semana, em que certamente formou um microclima com temperatura mais uniforme e os frangos adultos, devido ao menor espaço, caminharam menos no galpão, economizando energia.

Para a fase de 8 a 14, 29 a 35 e 36 a 42 dias de idade não foram verificadas diferenças significativas $(\mathrm{P}>0,05)$ entre os programas de alimentação e entre as densidades. De forma similar, não se constataram interações significativas entre programas de alimentação e densidades.

Observou-se que no período total, 1 a 42 dias de idade, não ocorreram diferenças significativas $(\mathrm{P}>0,05)$ entre programas de alimentação e densidades para ganho de peso. Da mesma forma, as interações entre programas de alimentação e densidades também não foram significativas. Contudo, o maior ganho de peso $(2447,5 \mathrm{~g})$ foi observado no programa 2, na densidade de 10 aves $/ \mathrm{m}^{2}$. Esses resultados foram coerentes com os descritos por MOUCHREK et al. (1992a, b). Por outro lado, GOLDFLUS et al. (1997a, b) e HELLMEISTER FILHO et al. (1998) encontraram diferenças signifi- cativas para ganho de peso de frangos de corte criados sob diferentes densidades.

Na Tabela 5 encontram-se os dados de conversão alimentar, apresentados pelas aves no período de 1 a 42 dias de idade, de acordo com o programa de alimentação e a densidade. Não se constataram diferenças significativas $(\mathrm{P}>0,05)$ entre os programas de alimentação para a conversão alimentar. As interações entre programas de alimentação e densidades também não foram significativas. Nas fases de 1 a 7 e 15 a 21 dias de idade foram verificadas diferenças significativas $(\mathrm{P}<0,05)$ entre as densidades na conversão alimentar. Os melhores índices de conversão alimentar foram alcançados nas densidades de 12 e 16 aves $/ \mathrm{m}^{2}$, respectivamente nas fases de 1 a 7 e 15 a 21 dias de idade, no programa 1 de alimentação.

Para os períodos de 8 a 14, 22 a 28, 29 a 35 e 36 a 42 dias de idade não se verificaram diferenças significativas $(\mathrm{P}>0,05)$ entre os programas de alimentação e entre as densidades para a conversão alimentar.

No período total, de 1 a 42 dias de idade, não ocorreram diferenças significativas $(\mathrm{P}>0,05)$ entre programas de alimentação e densidades para conversão alimentar. Na densidade de 12 aves $/ \mathrm{m}^{2}$ e no programa de alimentação 2, a conversão alimentar foi de 1,88.

Os pesos das aves ao abate e a produção em $\mathrm{kg} /$ $\mathrm{PV} / \mathrm{m}^{2}$, de acordo com o programa de alimentação e as densidades aos 42 dias de idade, são apresentados na Tabela 6. 
LANA et al.

Tabela 5 - Conversão alimentar dos frangos, nos períodos semanais e total (1 a 42 dias), de acordo com o programa de alimentação e densidade

Table 5 - Feed:gain ratio of broiler chickens in the weekly and total periods (1 to 42 days), according to feeding program and density

\begin{tabular}{|c|c|c|c|c|c|}
\hline \multirow[t]{2}{*}{$\begin{array}{l}\text { Período (dias) } \\
\text { Period (days) }\end{array}$} & \multirow[t]{2}{*}{$\begin{array}{c}\text { Programa de alimentação } \\
\text { Feeding program }\end{array}$} & \multicolumn{3}{|c|}{$\begin{array}{c}\text { Densidades } \\
\text { Densities }\end{array}$} & \multirow[t]{2}{*}{$\begin{array}{l}\text { Média } \\
\text { Average }\end{array}$} \\
\hline & & 10 & 12 & 16 & \\
\hline \multirow[t]{2}{*}{$1-7$} & P1 & $1,11^{\mathrm{b}}$ & $1,00^{\mathrm{a}}$ & $1,04^{\mathrm{a}}$ & 1,05 \\
\hline & P2 & $1,15^{\mathrm{b}}$ & $1,02^{\mathrm{a}}$ & $1,03^{\mathrm{a}}$ & 1,07 \\
\hline \multirow[t]{2}{*}{$8-14$} & P1 & 1,59 & 1,55 & 1,56 & 1,57 \\
\hline & P2 & 1,55 & 1,53 & 1,45 & 1,51 \\
\hline \multirow{2}{*}{$15-21$} & P1 & $1,48^{\mathrm{b}}$ & $1,42^{\mathrm{a}}$ & $1,41^{\mathrm{a}}$ & 1,44 \\
\hline & $\mathrm{P} 2$ & $1,48^{b}$ & $1,45^{\mathrm{a}}$ & $1,45^{\mathrm{a}}$ & 1,46 \\
\hline \multirow[t]{2}{*}{$22-28$} & $\mathrm{P} 1$ & 1,74 & 1,74 & 1,76 & 1,75 \\
\hline & $\mathrm{P} 2$ & 1,69 & 1,70 & 1,76 & 1,72 \\
\hline \multirow[t]{2}{*}{$29-35$} & $\mathrm{P} 1$ & 2,04 & 1,94 & 1,99 & 1,99 \\
\hline & $\mathrm{P} 2$ & 1,95 & 1,94 & 2,00 & 1,96 \\
\hline \multirow[t]{2}{*}{$36-42$} & $\mathrm{P} 1$ & 2,62 & 2,85 & 2,75 & 2,74 \\
\hline & $\mathrm{P} 2$ & 2,84 & 2,77 & 2,91 & 2,84 \\
\hline \multirow{2}{*}{$1-42$} & $\mathrm{P} 1$ & 1,90 & 1,89 & 1,90 & 1,86 \\
\hline & $\mathrm{P} 2$ & 1,92 & 1,88 & 1,91 & 1,90 \\
\hline
\end{tabular}

Médias seguidas de letras diferentes, na mesma coluna, diferem $(P<0,05)$ pelo teste Tukey.

Means followed by different letters, in the same column, differ $(P<.05)$ by Tukey test.

Não houve diferenças significativas $(\mathrm{P}>0,05)$ entre programas de alimentação para os pesos das aves ao abate e para produção em $\mathrm{kg} / \mathrm{PV} / \mathrm{m}^{2}$. Esses resultados estão de acordo com os descritos por BERTECHINI et al. (1991), SOUZA et al. (1993), STRINGHINI et al. (1997a) e SILVA JR. et al. (1999), que não encontraram diferenças significativas em frangos de corte criados nas densidades de 8 a 14 aves $/ \mathrm{m}^{2}$.

No entanto, constataram diferenças significativas $(\mathrm{P}<0,05)$ entre as densidades para a produção em $\mathrm{kg} /$ $\mathrm{PV} / \mathrm{m}^{2}$, sendo a maior produção $(37,670 \mathrm{~g})$ obtida na densidade de 16 aves $/ \mathrm{m}^{2}$. Estes resultados estão de acordo com os obtidos por GOLDFLUS et al. (1997b) e STRINGHINI et al. (1998), os quais observaram maior produção por unidade de área para aves criadas em maior densidade por metro quadrado.

Os pesos absoluto e relativo de carcaça, peito, coxas e sobrecoxas dos frangos, de acordo com o programa de alimentação e a densidade, aos 42 dias de idade, encontram-se na Tabela 7.

Não se verificaram diferenças significativas $(\mathrm{P}>0,05)$ entre programas de alimentação e entre densidades para os pesos absoluto e relativo de carcaça, peito, coxas e sobrecoxas. As interações entre programas de alimentaçãoe densidade também não foram significativas $(\mathrm{P}>0,05)$.

Os maiores pesos de carcaça $(2117,1 \mathrm{~g})$, peito $(522,9 \mathrm{~g})$, coxas $(267,9 \mathrm{~g})$ e sobrecoxas $(318,1 \mathrm{~g})$ e o
Tabela 6 - Pesos de frangos de corte (42 dias de idade) ao abate e produção em $\mathrm{kg} / \mathrm{PV} / \mathrm{m}^{2}$, de acordo com o programa de alimentação e a densidade

Table 6 - Weights at slaughter and production in $\mathrm{kg} / \mathrm{LW} / \mathrm{m}^{2}$ of broiler chickens (42 days old), according to feeding program and density

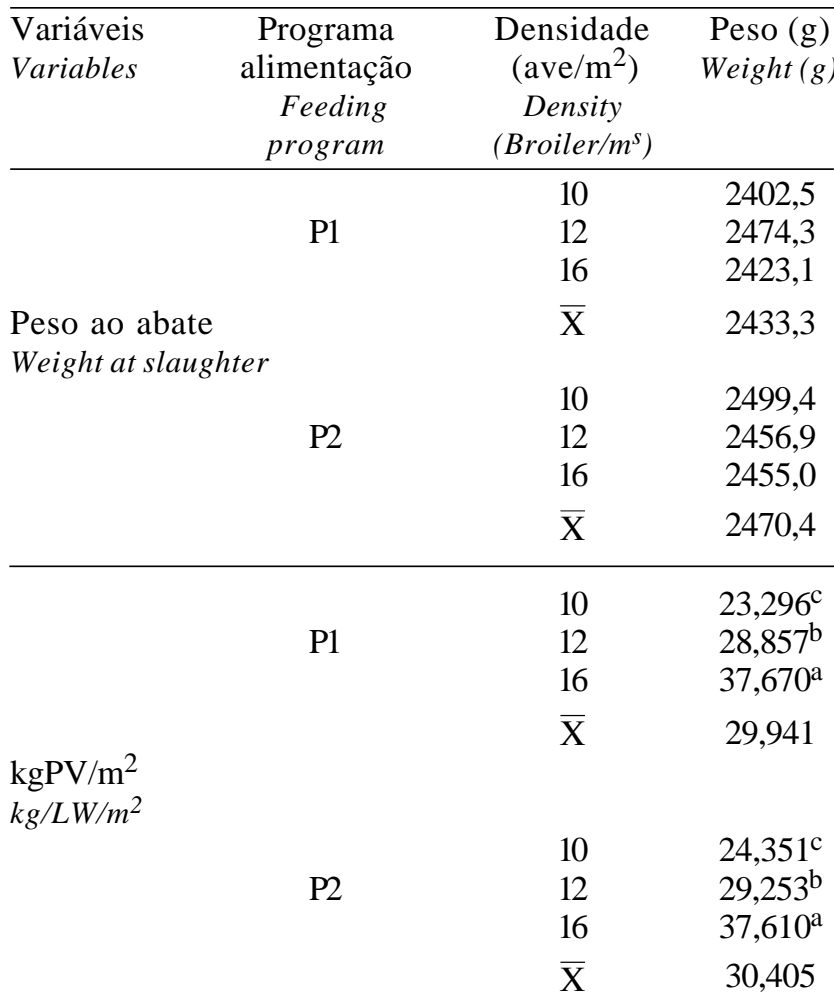

Médias seguidas de letras diferentes, na mesma coluna, diferem $(\mathrm{P}<0,05)$ pelo teste Tukey.

Means followed by different letters, in the same column, differ $(P<.05)$ by Tukey test. 
1264 Rev. bras. zootec.

Tabela 7 - Pesos absoluto (g) e relativo (\%) de carcaça, peito, coxas e sobrecoxas dos frangos, aos 42 dias de idade, de acordo com o programa de alimentação e a densidade*

Table 7 - Absolute $(\mathrm{g})$ and relative (\%) weights of carcass, breast, drumsticks and tights of broiler chickens at 42 days of age, according to feeding program and density

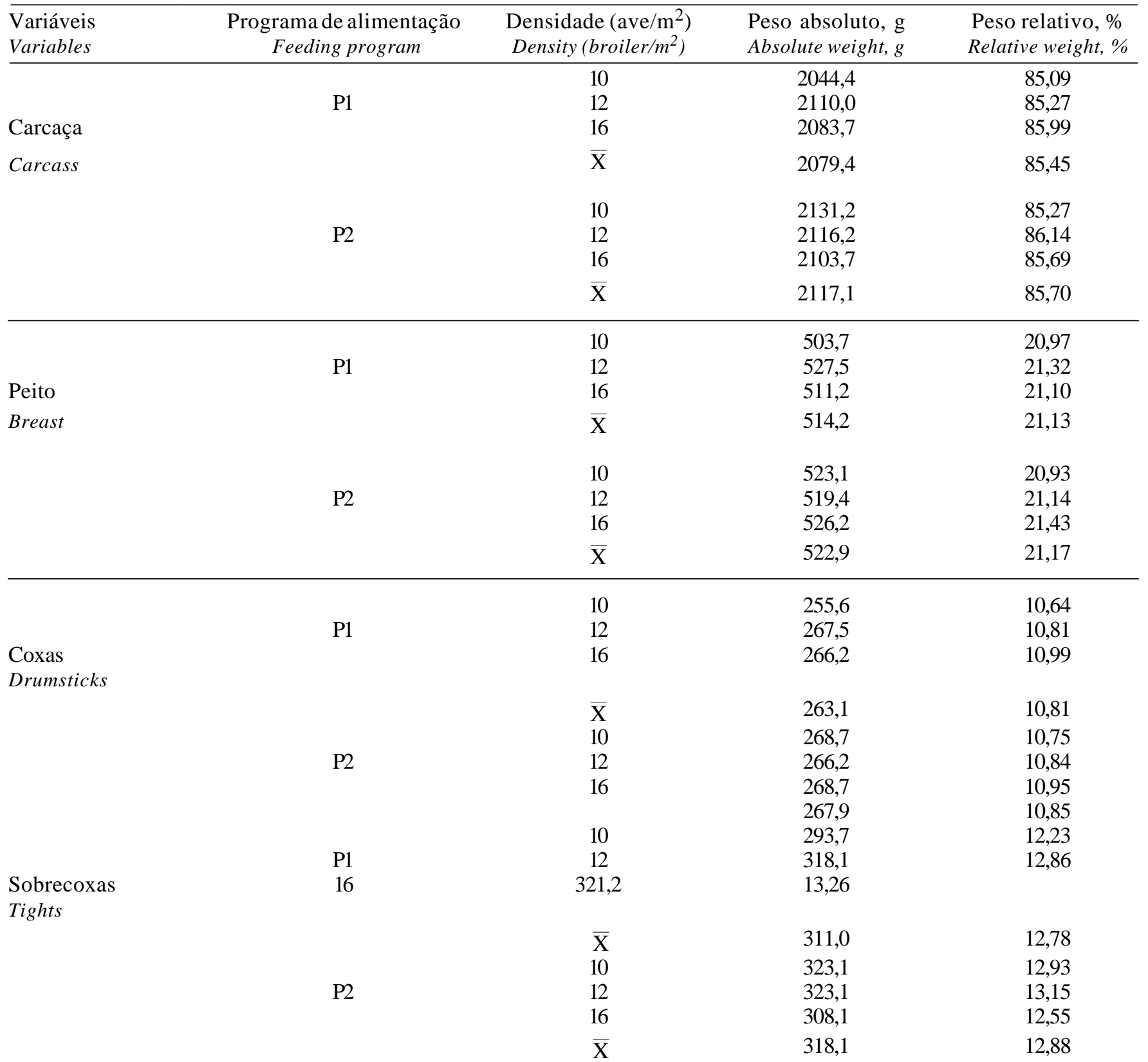

Não-significativo $(P>0,05)$ (Not significant $[P>05])$.

rendimento de carcaça $(85,70 \%)$, peito $(21,17 \%)$, coxas $(10,85 \%)$ e sobrecoxas $(12,88 \%)$ foram obtidos no programa 2. Com base nesses resultados, pode-se inferir que a utilização de três rações com níveis diferenciados de energia e proteína no período de 1 a 21 dias influenciou os pesos e rendimentos de carcaça e cortes nobres (peito, coxas e sobrecoxas), mostrando, assim, a grande importância da adoção de maior número de rações na criação de frangos de corte.

\section{Conclusões}

Recomenda-se para frangos de corte, machos, no período de 1 a 42 dias de idade, a densidade de 16 aves $/ \mathrm{m}^{2}$, independentemente do programa de alimentação, para se obter melhor desempenho produtivo das aves, desde que haja bom controle do ambiente e adequado manejo alimentar. 


\section{Referências Bibliográficas}

ABREU, P.G. 1999. Ambiência avícola. Revista Avicultura Industrial, São Paulo, (1070):18-22.

BERTECHINI A.G., ROSTAGNO, H.S., SOARES, P.R. et al. 1991. Efeitos de programas de alimentação sobre o desempenho e a carcaça de frangos de corte. Rev. Soc. Bras. Zootec., 20(3):267-280.

GOLDFLUS, F., ARIKI, J., KRONKA, S.N. et al. 1997a. Efeitos da densidade populacional e da energia da dieta sobre o desempenho de frangos de corte. Rev. Soc. Bras. Zootec., 26(2):310-215.

GOLDFLUS, F., ARIKI, J., KRONKA, S.N. et al. 1997b. Efeitos de diferentes densidades populacionais nas estações fria e quente do ano sobre o desempenho de frangos de corte. Rev. Soc. Bras. Zootec., 26(5):948-954.

HELLMEISTER FILHO, P., CUSTÓDIO, R.W.S., COELHO, A.A.D. et al. 1998. Desempenho de frangos de corte criados em diferentes densidades. R. Soc. Bras. Zootec., 27(1):137142.

LANA, G. R. Q. 2000. Avicultura. 1.ed. Campinas: Livraria e Editora Rural Ltda. 268p.

LUCCHESE FILHO, A. Criação de frangos de corte em alta densidade: pré requisitos, vantagens e desvantagens do sistema. In: MANEJO DE FRANGOS DE CORTE, 1997, Campinas. Anais... Campinas: FACTA, 1997.p.13-22.

MOUCHREK, E., TOSHIYUKI, T., STHLING, R. et al. Desempenho de uma linhagem de frangos de corte submetida a diferentes densidades populacionais na época quente. In: REUNIÃO ANUAL DA SOCIEDADE BRASILEIRA DE ZOOTECNIA, 29, 1992, Lavras. Anais... Botucatu: SBZ, 1992a. p.345.

MOUCHREK, E., TOSHIYUKI, T., STHLING, R. et al. Desempenho de uma linhagem de frangos de corte submetida a diferentes densidades populacionais na época fria. In: REUNIÃO ANUAL DA SOCIEDADE BRASILEIRA DE ZOOTECNIA, 29, 1992, Lavras. Anais... Botucatu: SBZ, 1992b. p.346.

MUNARI, J.L.P. Criação de frangos em alta densidade: vantagens e desvantagens. In: TÓPICOS ATUALIZADOS NA PRODUÇÃO DE FRANGOS DE CORTE, 1997, Uberlândia. Anais... Uberlândia: UFU, 1997. n.p.

ROSTAGNO, H.S., BARBARINO JR., P., BARBOZA, W. A. Exigências nutricionais das aves determinadas no Brasil. In: SIMPÓSIO INTERNACIONAL SOBRE EXIGÊNCIAS NUTRICIONAIS DE AVES E SUÍNOS, 1996, Viçosa. Anais... Viçosa: UFV. p.361.

SHIROMA, N.N., ZANETTI, M.A., FARIA, D.E. et al. Efeito de programas de alimentação sobre o desempenho de frangos de corte machos durante o inverno. In: CONFERÊNCIA APINCO DE CIÊNCIA E TECNOLOGIA AVÍCOLAS, 1996, Curitiba. Anais... Campinas: FACTA, 1996. p.28.
1265

SILVA JR., R.G.C., LANA, G.R.Q., CORDEIRO, E.C.G.B Avaliação de dois programas de alimentação e do sexo sobre o desempenho e rendimento de cortes nobres em frangos. In: REUNIÃO ANUAL DA SOCIEDADE BRASILEIRA DE ZOOTECNIA, 36, 1999, Porto Alegre. Anais... Botucatu: SBZ, 1999. p.344.

SIMON, V.A. Aspectos sanitários de criações em altas densidades. In: SIMPÓSIO SOBRE AMBIÊNCIA, SANIDADE E QUALIDADE DE CARCAÇA DE FRANGOS DE CORTE, 1997, Concórdia. Anais... Concórdia:EMBRAPACNPSA, 1997. p.14-18.

SOUZA, P. A., SOUZA, H. B. A., BROGNONI, E. et al. Desempenho e características de carcaças de diferentes linhagens comerciais de frangos de corte. In: CONFERÊNCIA APINCO DE CIÊNCIA E TECNOLOGIA AVÍCOLAS, 1993, Santos, Anais... Campinas: FACTA, 1993. p.84.

STRINGHINI, J.H., ARIKI, J., CAFÉ, M.B. et al. Níveis de proteína para frangos de corte criados em duas densidades populacionais, II. Características de carcaça. In: CONFERÊNCIA APINCO DE CIÊNCIA E TECNOLOGIA AVÍCOLAS, 1997, São Paulo. Anais... Campinas: FACTA, 1997a. p.24.

STRINGHINI, J.H., ARIKI, J., CAFÉ, M.B. et al. Níveis de proteína para frangos de corte criados em duas densidades populacionais, I. Desempenho. In: CONFERÊNCIA APINCO DE CIÊNCIA E TECNOLOGIA AVÍCOLAS, 1997, São Paulo. Anais... Campinas: FACTA, 1997b. p.25.

STRINGHINI, J.H., ARIKI, J., CAFÉ, M.B. et al. Níveis de lisina para frangos de corte criados em duas densidades populacionais. In: REUNIÃO ANUAL DA SOCIEDADE BRASILEIRA DEZOOTECNIA, 35, 1998, Botucatu. Anais.. Botucatu:SBZ, 1998. p.216.

UNIVERSIDADE FEDERAL DE VIÇOSA-UFV 1982. SAEG Sistema de Análises Estatísticas e Genéticas. Viçosa, MG. 59p. (Manual do usuário).

Recebido em: 20/11/00 Aceito em: 06/03/01 